\title{
Rapid, Automated Tissue Processing Techniques for Small Biopsies Can be used to Produce Quality Sections for Diagnostic Reporting in Histopathology
}

\author{
Finall AI*, Parris 0 and Jones E
}

Department of Cellular Pathology, Swansea Bay University Health Board, Morriston Hospital, UK

*Corresponding author: Alison Finall, Consultant Histopathologist, Swansea Bay University Health Board Honorary Associate Professor, Swansea University, Tel: 01792 702222; Email: alison.finall3@wales.nhs.uk

\section{Research Article}

Volume 3 Issue 1

Received Date: March 06, 2020

Published Date: May 29, 2020

DOI: $10.23880 /$ aabsc-16000148

\section{Abstract}

Surgical histopathologists are currently working in a context of high demand and requirement for fast turnaround times to report small diagnostic biopsies. Use of automated rapid cycle processing could improve turnaround times for reporting small biopsies. There are few papers in the medical literature describing detailed assessment of staining quality in verification of automated rapid cycle processing methods.

This study assessed quality of immunohistochemical and haematoxylin and eosin staining of 62 paired endometrial biopsy samples processed using standard overnight cycles compared with rapid cycles of either 2, 3 or 4 hours duration. We found that small biopsies adequately fixed in $10 \%$ neutral buffered formalin were of sufficient staining quality for use in diagnostic reporting if they were processed for 3 or 4 hours. Five samples in our 2-hour group were of insufficient staining quality for full microscopic evaluation and histopathological diagnosis. As such, the 2-hour method was rejected. Rapid cycles of three hours duration will be adopted in our laboratory for processing small samples. Prospective monitoring of report turnaround times will be needed to assess impact on future outcomes for patients.

Keywords: Histopathologists; Immunohistochemical; Haematoxylin; Impregnantion

\section{Introduction}

\section{Problem Description}

The workloads of both histopathologists and diagnostic laboratories is increasing due to a greater number of samples of increasing complexity in an era of personalised medicine. ${ }^{1}$ This has the potential to increase turn around times for patient histopathology reports [1]. It is not unusual for a histopathologist to be asked for a report by clinicians before they have received the slides from the laboratory. Diagnostic histopathology reports rely upon production of high-quality glass slides for microscopic interpretation by a either qualified advanced biomedical scientist (BMS) or, more commonly, a consultant histopathologist. Automated overnight batch processing is typically used to process formalin fixed tissue samples into paraffin embedded blocks ready for cutting into sections for glass mounted slides. The introduction of paraffin into the tissues to replace water and fat is required to make the tissue firm enough to cut into 4-5 micrometre thick sections for staining on glass slides $[2,3]$. This processing technique is usually automated in large hospital laboratories and requires cycles of immersion in alcohol and xylene to dehydrate and clear the tissue respectively. These sections are then stained with standard haematoxylin and eosin (H\&E) to highlight cellular detail for interpretation; haematoxylin preferentially highlights nuclei and eosin stains cytoplasm [2,3]. In addition, some 
cases of poorly differentiated malignancy for example, require immunohistochemistry for ancillary information to aid diagnostic decision-making particularly in a context of metastatic disease.

A typical automated processing protocol requires dehydration steps of $1 \frac{1}{2}$ hours each in increasing concentrations of isopropyl alcohol at $60 \%, 70 \%, 80 \%$, and $90 \%$. This is followed by two $1 \frac{1}{2}$ hour steps in $100 \%$ alcohol alternating with xylene over 4 steps of 1 hour followed by impregnantion with paraffin for up to 4 hours. The total time for a standard automated cycle is 17 hours [2]. Standard processing cycles are most frequently done by batching cases for overnight processing ready for embedding into paraffin blocks the following morning. Often laboratories will have a processor standing unused during the day as a consequence of this workflow pattern.

Some urgent cases that are suspected of harboring malignancy are required as soon as possible and for discussion at multi-disciplinary team meetings (MDT) to plan patient treatment. Often these urgent cases are small tissue biopsies that are suitable for processing using rapid cycles of between 2 and 4 hours, thus reducing the time to make slides available to the pathologist for reporting and making it more likely that a diagnosis can be offered in time for discussion at MDT. They are suitable for rapid processing cycles as the small amount of tissue allows for shorter fixation times in $10 \%$ neutral buffered formalin. Formalin fixation occurs at a tissue penetration rate of $1 \mathrm{~mm}$ per hour [4]. Formalin fixation is required to prevent tissue degradation.

This is a validation study to assess quality of $H \& E$ and immunohistochemical staining using rapid cycle processing as compared with standard overnight cycles. Verification projects such as this are required to show quality compliance to standards set out in ISO15189 for medical laboratory accreditation.

\section{Available Knowledge}

There are few papers in the medical literature describing verification of automated short processing cycles in the histopathology laboratory. A study by Jali, et al. [5] assessed a rapid manual technique for use in areas of the world without reliable continuous electricity supply and access to automation. Their manual test protocol was 8 hours long to fit in a normal working day after a 15 -hour overnight step in $60 \%$ alcohol. They found no difference between their rapid cycle and the standard automated protocol when assessing for tissue shrinkage [5]. There was a difference of $4.4 \%$ for staining quality assessment across the two schedules. Interestingly, $20 \%$ of their rapid cycle slides had short comings compared with $24.4 \%$ of the standard cycles [5].
They do not state whether this difference was statistically significant or whether any of the shortcomings were likely to affect the ability of the pathologist to make a diagnosis. An interesting study by Morales, et al. in 2002 [6] describes a novel processing method using mineral oils and solutions of combinations of alcohol, polyethylene glycol, dimethyl sulfoxide, acetone and glacial acetic acid for use on fresh large and small samples. They found that fresh tissue processing in samples less than $3 \mathrm{~mm}$ thick can be processed in around 1 hour using their method. They also claim that the resulting paraffin-embedded tissue blocks are less brittle than formalin fixed tissue blocks and easier to cut into sections at the microtome. They state that this formalin-free method produced H\&E sections requiring half the concentration of H\&E stain to achieve comparable sections but the details of how staining quality was assessed is not clearly indicated. Morales, et al. declare experience of conventional automated rapid tissue processing cycles of up to 2.5 hours duration and state this is only suitable for small samples because of formalin fixation requirements [6]. However, staining quality from the 2.5 hour cycles is not discussed. Tissue processors using microwave energy and variable increased pressure to reduce processing time are described [7]. Other studies have focused on rapidity of fixation methods using microwave energy to improve turnaround times for producing histopathology slides [8].

\section{Rationale}

Any change in process adopted by a histopathology laboratory needs to be internally validated to meet medical laboratory standards for accreditation. This study aimed to validate the method of short cycle processing in the histopathology laboratory as being as good as standard overnightcyclesforquality ofH\&Eandimmunohistochemistry staining. The rationale is that producing slides in a shorter time frame would have a positive impact on turnaround times for histopathological reporting. This could then improve the patient experience by having earlier treatment interventions based on the diagnosis. Shorter processing cycles could also mean use of processes at multiple time points during the day when the processors would otherwise be standing idle. This is a more efficient approach to laboratory workflow with reduced case batching. It may, however, require BMS and medical laboratory assistant (MLA) staff time.

\section{Specific Aims}

The purpose of the project was to determine whether small biopsy tissue section staining quality with H\&E and immunohistochemistry was affected by automated short cycle processing. Specifically, we needed to know whether the resulting sections could be reliably used for histopathological diagnosis. 


\section{Methods}

\section{Context}

This study aims to validate tissue section staining quality in a diagnostic clinical setting in accordance with laboratory accreditation standards (ISO 15189). This evidence must be generated before any change in laboratory process is implemented.

Our laboratory staff is committed to producing highquality sections for diagnosis and to rapid turnaround for urgent samples in the interests of planning treatment for patients, particular those with cancer. Timely diagnosis of cancer can impact on disease free and overall survival of patients across the spectrum of cancers. Lung cancer is a good example of this where it has been shown that a $10 \mathrm{~mm}$ increase in diameter of a malignant tumour mass correlates with a $10 \%$ reduction in overall five year survival for early stage cancers of TNM stage $1[9,10]$.

\section{Intervention}

A randomized, prospective, consecutive cohort of 62 paired endometrial biopsy samples was included in the project following receipt in the laboratory. Endometrial samples were selected as they could be split into two equal halves for processing on a standard overnight cycle and a rapid cycle of either 2, 3 or 4 hours using Thermoscientific Exselsior ES processors (2008). The difference between each of these cycles results in a reduction of proportional amounts of time in formalin fixation, alcohol dehydration, xylene clearing and paraffin embedding according to manufacturers instructions. Using different techniques on the same sample allowed standard processes to continue in parallel for every patient thus ensuring no diagnostic compromise. As this is a study of quality of staining rather than morphological diagnosis it was not deemed necessary to have a sample size large enough to capture all potential diagnostic entities relevant to the tissue type.

Samples that were not fixed adequately according to the professional judgment of biomedical scientist (BMS) staff were excluded. Samples too small to be split across two tissue cassettes were also excluded to preserve diagnostic material and ensure no patient samples were compromised.

All endometrial biopsies were assessed by a senior BMS. If suitable, the biopsy was split into two cassettes labeled A1 and A2 for allocation to standard processing or rapid processing. Cassette allocation data was recorded by the senior BMS. This information was not available to view by the advanced reporting BMS or the supervising pathologist at the time of assessment to ensure blinding to protocol was maintained during quality scoring. The rapid cycle slide was kept back in the laboratory until its partner had finished processing by standard overnight protocols so they could both be presented for reporting at the same time with the clinical request form.

Both A1 and A2 H\&E slides and request form were given to the reporting BMS for provisional reporting and then sent to the supervising pathologist for checking and report authorisation. The supervising pathologist requested vimentin (cytoplasmic immunohistochemistry (IHC) stain), EMA (a membranous IHC stain) and PR (a nuclear IHC stain) on both $\mathrm{A} 1$ and $\mathrm{A} 2$ for comparison of quality. The choice of additional immunohistochemistry had no potential diagnostic impact and was selected to represent each of the cell compartments nucleus, cytoplasm and membrane for a full assessment of quality. Vimentin is considered a useful marker of adequate tissue preservation and processing $[11,12]$.

Ten slides were generated for each patient sample; five from the rapid cycle, five from the standard cycle as follows: $1 \mathrm{H} \& \mathrm{E}$ and 4 IHC slides. Each of the investigators made an independent comparative assessment of the staining quality of each of the slides using and agreed and clearly described scoring algorithm based on industry standards $[12,13]$.

\section{Study of the Interventions}

The perception of quality was tabulated as a numerical score according to standardized criteria. Data were compared between the two reporters advanced BMS and clinical histopathologist. Cases with disagreement were discussed and reviewed together over a multi-headed microscope. A consensus was reached through detailed case review and discussion between the three investigators. Cases where staining was not of sufficient quality for clinical diagnostic reporting were particularly highlighted. Consensus scores were tabulated and correlated with the original assignation to standard or rapid cycle process. An overall dichotomous assessment of whether the features were suitable for clinical diagnosis was made for each sample. It was considered that any failure in this category would adversely affect any decision to adopt a rapid cycle processing of particular time duration. One failure would be sufficient to disqualify an enhanced cycle of any time interval from consideration as a practical option for improving slide production turnaround time.

\section{Measures}

H\&E quality was scored according to established criteria with respect to tissue preservation and staining appearances as follows [13]. 
Tissue preservation: nuclear detail (good $=2$, fair $=1$, poor $=0$ ), cytoplasmic detail (good $=2$, fair $=1$, poor $=0$ ), uniformity of preservation (good $=2$, fair $=1$, poor $=0$ ),

Quality of staining: nuclear stain (good $=2$, fair $=1$, poor $=0$ ), cytoplasmic stain (good $=2$, fair $=1$, poor $=0$ ), uniformity of staining (good $=2$, fair $=1$, poor $=0$ ).

Quality of immunohistochemical staining was scored as follows [12]:

Staining intensity (Score as follows: no staining $=3$, weak staining $=2$, overstained $=1$, ideal staining $=0$ );

Uniformity of staining (Edge artifact=2, gradient staining=1, good uniformity across section $=0$ )

Specificity (Incorrect cellular compartment=1, correct compartment $=0$ )

Background ( macrophage $=2$, red cell staining $=1$, none $=0$ )

Counterstaining (no counter stain $=3$, gradient staining $=2$, over-stained $=1$, ideal counterstain $=0$ ).

The consensus scores for each tissue sample were tabulated as a percentage of a possible perfect score of 100 on an excel spread sheet for comparative analysis.

\section{Analysis}

Comparative analysis of tabulated consensus outcome quality scores was made with reference to the processing cycle status of each slide set. Statistical significance was tested using Chi square test for categorical data. Probability scores less than or equal to $5 \%(p=<0.05)$ was considered statistically significant.

\section{Ethical Considerations}

This was an internal validation study of processing methods within the histopathological laboratory to fulfill the criteria of ISO15189 requirements for quality and competence in medical laboratories. The study had no impact on diagnostic material or patients. Patient samples too small to be split across two tissue cassettes were excluded from the study. Patient consent was not required. All information generated from immunohistochemical analysis was reported alongside morphological data and the markers selected had no effect on the diagnostic outcome for this tissue and biopsy type.

\section{Results}

Thirteen cases had to be excluded from analysis for not having sufficient cellular material on the glass slides to give an accurate microscopic score leaving 49 cases for assessment. There were 14 cases in the 3 -hour and 4-hour group each and 21 cases in the 2-hour group. Consensus scores were tabulated as an average between pathologist and advanced reporting BMS scores. There was no statistical significance between the scores from pathologist and BMS ( $p>0.05)$.

\begin{tabular}{|c|c|c|c|}
\hline \multicolumn{4}{|c|}{ Consensus outcomes for slide quality (H\&E) } \\
\hline Cycle & Number of Tests & Number of Passes & Average Score (\%) \\
\hline 2 hour & 21 & 16 & 82.8795 \\
\hline routine & 21 & 21 & 96.2798 \\
\hline 3 hour & 14 & 14 & 89.28571 \\
\hline routine & 14 & 14 & 96.42857 \\
\hline 4 hour & 14 & 14 & 93.75 \\
\hline routine & 14 & 14 & 95.75893 \\
\hline
\end{tabular}

Table 1: Consensus outcomes and average score totals for H\&E quality of two independent assessors.

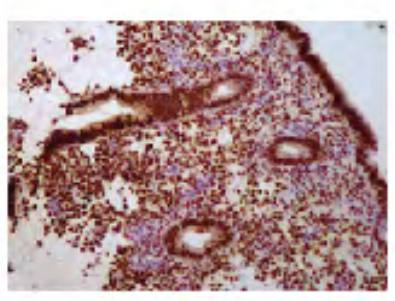

1A

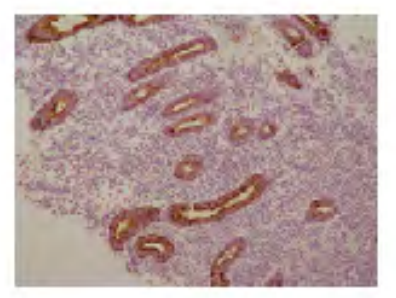

1B

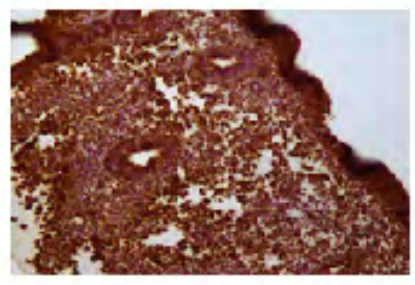

$1 \mathrm{C}$

Figure 1: Immunohistochemistry examples from across the data set were satisfactory. $1 \mathrm{~A}$ is progesterone receptor, 1B EMA and $1 \mathrm{C}$ vimentin demonstrating nuclear, membranous and cytoplasmic reactivity respectively. Photomicrographs taken at x10 magnification. 
All the IHC slides reviewed were of sufficient quality for diagnosis across all cycle durations. There was no difference in scores between standard cycles and short cycles $(2,3$ and
4 hours) for staining quality scores for vimentin, EMA and PR (Figure 1).

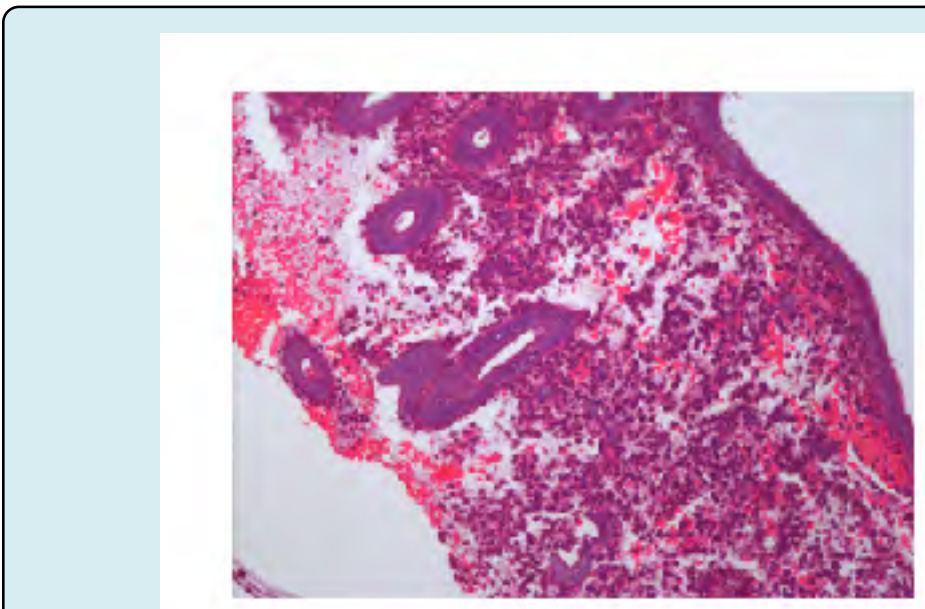

$2 \mathrm{~A}$

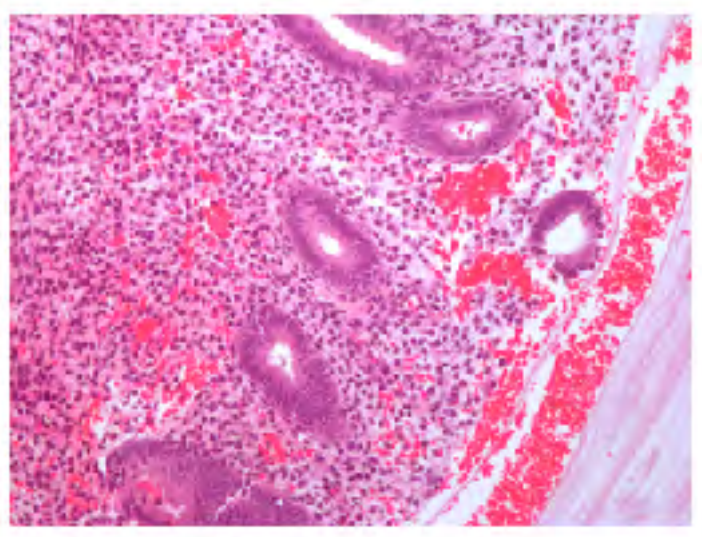

$2 B$

Figure 2A: Photomicrograph of endometrial biopsy processed using a rapid 2-hour cycle that shows diffuse blue artefact. The blue artefact blurs nuclear cytoplasmic contrast and causes a loss of chromatin detail that can hamper cytological assessment of dysplasia. Figure 2B: Standard overnight cycle of endometrial biopsy for comparison. Both stained with H\&E and viewed at x10 magnification.

Overall, there were no slides in the 3- or 4-hour processing group that were considered of insufficient quality to potentially hamper diagnosis. The H\&E scores were expressed as a percentage of a total $100 \%$ perfect possible score. The average H\&E quality score for the 4-hour group was $93 \%$. The average H\&E quality score for the 3-hour group was $89.5 \%$. The average H\&E quality score for the 2 -hour group was $83.5 \%$. Five H\&E slides from the 2-hour group were considered of poor quality due to diffuse blue artifact (Figure 2A and 2B). The artifact, with loss of nuclearcytoplasmic contrast and chromatin detail, could interfere with assessment for cytoplasmic atypia as defined for atypical hyperplasia of the endometrium. One failure was all that was required to discount a short cycle period as a potential option for processing patient samples. In addition the difference between scores in the 2-hour group and expected scores was statistically significant $(\mathrm{p}=0.017)$.

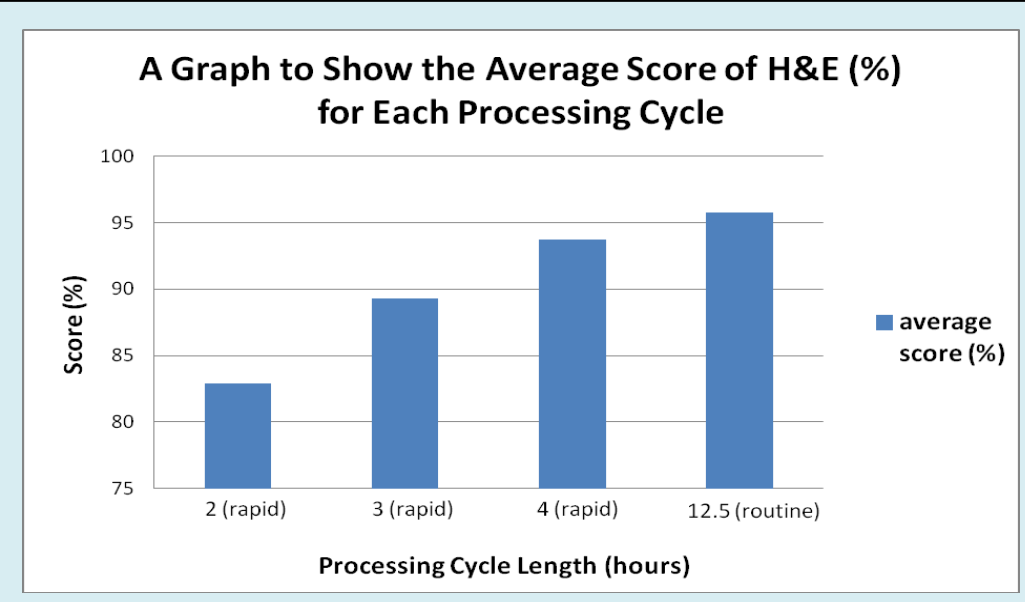

Chart 1: Average quality score for H\&E slide for each time period. 2, 3 and 4 hours are defined as rapid or short processing cycles. Routine or standard automated tissue processing takes 12 hours. 


\section{Discussion}

\section{Summary}

Overall, our findings support the use of rapid cycle processing of endometrial biopsies that are adequately fixed in $10 \%$ neutral buffered formalin and undergo processing for 3 or 4 hours.

\section{Interpretation}

Five of 21 samples processed on 2-hour cycles generated diffuse blue artefact that blurs the interface between nucleus and cytoplasm within cells and obscures chromatin pattern within the nucleus itself. The cloudiness of diffuse blue artifact is said to arise from defective dehydration during processing that can requires reprocessing for correction. ${ }^{14}$ Diffuse blue artifact hampers the ability to identify features of cytological atypia in endometrial hyperplasia. Diagnosis of atypical hyperplasia of the endometrium is an important diagnosis as it is a pre-cursor for endometrioid-type endometrial adenocarcinoma and may also be a trigger for treatment depending upon the clinical context of the patient. Assessment of cytological atypia is based on morphological appearances at a microscopic level and requires highquality H\&E staining. The 2-hour duration rapid cycles were excluded from options appraisal for service improvement in our laboratory.

It is well known that length of exposure to formalin can effect the quality of IHC staining by standardised protocol [11]. We use heat retrieval in our laboratory to undo formalin cross links that prevent tissue degradation but mask antigens for staining in the IHC antibody reaction. Tissue that is not adequately fixed can generate false positive IHC results as a consequence. Standard IHC protocols recommended 6-72 hours in formalin for optimum staining. All samples submitted for the study were assessed for adequate fixation prior to entry into the study and were fixed for at least 6 hours. The formalin exposure reduction in the shorter cycles is reduced by 30 minutes compared with a standard overnight cycle. Because all the samples were appropriately fixed before processing there was no effect on quality of immunohistochemistry staining in any of the rapid cycle groups. Vimentin staining was consistently good across the slide set and this supports the BMS assessments that tissue was adequately fixed prior to entry into the study [11].

\section{Limitations}

The study methodology relies upon meaningful and accurate conversion of morphological perceptions regarding tissue staining into a score for analysis. The scoring algorithm used was based upon evidence in the literature generated by groups involved in validation of high-quality automated systems in industry $[12,13]$. Despite the use of a robust scheme, there remains the possibility of subjective variation between observers in interpretation of the cytological features seen. Consensus discussion of cases where there was disagreement in scoring overcomes the limitation of interobserver variation. The pathologist and reporting BMS were blind to block processing protocol time and this enhances the reliability of the data generated through reduction of internal perception biases.

Our validation findings apply only to endometrial samples. It could be reasonably suggested that testing other tissue types may result in different findings. Typically endometrial samples contain a lot of blood and this may yield a different result compared with, for example a punch biopsy of skin, which is usually accompanied by very little blood. The applicability of the findings of this study should be considered limited to endometrial biopsy processing in our laboratory. Every medical laboratory needs to internally verify their processes according to IS015189 for accreditation purposes. There may be difficulty in splitting small tissue biopsies of differing tissue types for parallel processing of differing lengths of time. One may need to use a surrogate for small skin punch biopsies for example by using punch biopsies donated from larger excisions where there is considerable background material not required for diagnosis. Repetition of this work across all sample types would have significant resource implications for histopathology laboratories in terms of cost, staffing and time.

\section{Conclusions}

We conclude that rapid cycle processing of endometrial biopsies of 3 or 4 hours duration can yield the same highquality staining profile as that generated by standard overnight automated processing. Implementation of rapid cycles in our laboratory has the potential to reduce turnaround times for reporting small, urgent diagnostic biopsies that are often required for MDT in the care of patients with cancer.

\section{Funding}

This work received no external funding. The costs of additional IHC and additional slide production were borne by the department as a necessary investment in laboratory services. Reduced reporting time may yield downstream cost savings in terms of improved cancer survival outcomes, reduced hospital stay and reduced incidence of litigation. The authors declare an interest in developing diagnostic services to the highest standards of quality and safety in the best interests of patient care. The authors declare no conflicts of interest. 


\section{Author Contributions}

Dr Alison Finall conceived the project, designed the validation study, made slide quality assessments, participated in consensus review and wrote the paper. $\mathrm{Mr}$ Owen Parris, biomedical scientist, collected and analysed data and participated in consensus review and reviewed the manuscript. Mr Eilir Jones, Advanced Practitioner Histopathology, made slides quality assessments, participated in consensus review and reviewed the manuscript

\section{References}

1. Cancer Research (2019) Testing times to come? An evaluation of pathology capacity across the UK.

2. Ganjali H (2012) Tissue processing: An overview. Annals of Biological Research 3(11): 5374-5378.

3. Bancroft JD, Gamble M (2008) Theory and practice of histological techniques, ${ }^{\text {th }}$ (Edn.), Churchill Livingstone, Edinburgh.

4. Medawar PB (1941) The rate of penetration of fixatives. J Royal Micro Soc 61: 46-57.

5. Jali P, Donohue M, Gadiwan M (2015) A rapid manual processing technique for resource limited small laboratories. J Oral Maxfac Pathol 19(3): 306-314.

6. Morales AR, Essenfeld H, Essenfeld E, Duboue MC, Vinek V, et al. (2002) Continuous-Specimen-Flow, Highthroughput, 1 hour tissue processing. A system for rapid diagnostic tissue preparation. Arch Pathol Lab Med
126(5): 583-590.

7. Visinoni F, Milios J, Leong AS-Y, Boon ME, Kok LP, et al. (1998) Ultra-rapid microwave/variable pressure induced histoprocessing: description of a new tissue processor. J Histotechnol 21(3): 219-224.

8. Tripathi M, Bansal R, Gupta M,Vinay B (2013) Comparison of routine fixation of tissues with rapid tissue fixation. J Clin Diagn Res 7(12): 2768-2773.

9. Motta G, Carbone E, Spinelli E, Nahum MA, Testa T, et al. (1999) Considerations about tumor size as a factor of prognosis in NSCLC. Ann Ital Chir 70(6): 893-897.

10. Gajra A, Newman N, Gamble GP, Abraham NZ, Kohman LJ, et al. (2003) Impact of tumor size on survival in stage IA non-small cell lung cancer: a case for subdividing stage IA disease. Lung Cancer 42(1): 51-57.

11. Battifora H (1991) Assessment of antigen damage in immunohistochemistry: the vimentin internal control. Am J Clin Pathol 96(5): 669-671.

12. Lin F, Zongming C (2014) Standardization of Diagnostic immunohistochemistry. Arch Pathol Lab Med 138(12): 1564-1577.

13. Rolls G, Farmer N, Tarbet F (2008) Assessing the quality of tissue processing and the performance of Peloris using the Leica microsystems scoring system. Pub. Leica Microsystems GmBH.

14. Chatterjee S (2014) Artefacts in histopathology. J Oral Maxfac Pathol 18(S1): S111-S116. 